[Technical Paper]

\title{
Issues and Approaches Imposed on Ink Jet Technologies for the Progress of Printed Electronics
}

\author{
Masahiko Fujii \\ Ink jet Technology Laboratory, Corporate Research \& Technology Development Group, Fuji Xerox Co., Ltd., 2274 Hongo Ebina, Kanagawa 243-0494, \\ Japan
}

(Received August 5, 2010; accepted September 30, 2010)

\begin{abstract}
The simplicity of the ink jet process has led to major changes in electronics packaging in what is known as printed electronics. Major changes here means drastic cost reduction relative to the long-term trend, and also creating a new performance axis (flexibility, customization, large area, little environmental load ... namely added value). However, simplicity has two sides: 'Possibility' and 'Limitation.' For the past several years, 'Possibility' has been attracting a lot of attention, but in the coming years, if ink jet technology does not overcome 'Limitation', the real change will never occur.

In this paper, the related issues and status of approaches resolved by ink jet printheads or the printing process are described for the further progress and practical application of printed electronics using ink jet technologies.

These issues can be categorized into 1) ejecting processes of functional liquid, 2) drop landing and dot (film) formation on the substrate, and 3) maintaining printhead reliability. In particular, expanding the range of applicable liquids, further miniaturization of the ejected liquid drop, and improved accuracy of dot positioning are strongly required for printheads. In printed electronics, unlike with desktop ink jet printers, impermeable media are often used, so analysis and control of the behavior of the liquid on the substrate are also important. Furthermore, technologies for maintaining stability in the ejected drops make a difference.

These novel activities for improving ink jet issues will face tough problems and need revolutionary approaches, but a great innovation in printed electronics using ink jet technologies can be expected when success is achieved.
\end{abstract}

Keywords: Ink Jet, Printed Electronics, Drop Volume, Liquid Latitude, Viscosity, Airflow, Self-Alignment

\section{Introduction}

Applications of ink jet technologies outside of printing equipment have been investigated for a long time because of the simplicity of the ink jet process. In the past ten years, nobody has doubted that printing electronics using ink jet technologies is one of the most suitable applications to develop the characteristics of ink jet to their fullest.

Figure 1 shows a Portfolio of applications producing value by adopting ink jet technology, categorized by the features of ink jet they require.[1] As shown in this figure, applications in various fields, including printed electronics, have been investigated and developed. However, in spite of these efforts, most of the applications have neither appeared in our ordinary life nor yet been successful in business, with a few exceptions.

There is a famous quote representing the gap between the possibility and the reality (the limitations of ink jet) in the Printed Electronics region.

"Ink jet can make everything, but have manufactured

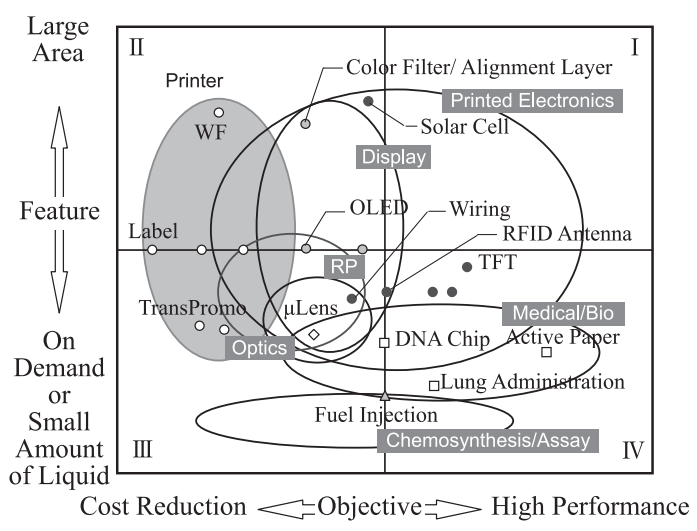

Fig. 1 Application of ink jet to digital fabrication.[1] 
nothing."

To solve this riddle and bring printed electronics using ink jet technology to a successful implementation, it is necessary to know what has prevented its realization and how we should tackle it technically. So in this paper, I will summarize the issues with and current approaches to this application of ink jet technology.

Shimoda categorized digital fabrication using ink jet into four processes and explained contents.[2] I would like to focus on the issues and approaches regarding ink jet printheads and review them based on my long experience in ink jet research and development.

\section{Liquid Ejection Process}

Ink jet can be classified by the type of actuator, namely how it gets the energy to generate and eject liquid drops,[3] and many kinds of ink jet have been realized. Piezo Ink Jet (PIJ), utilizing the displacement of lead zirconate titanate (PZT), is the type mainly employed in printed electronics, and a commodity-type printhead for commercial printers is used in most situations. The printed electronics market is not currently large enough to develop customized printheads, so there is no choice but to use a commodity printhead. However, the limited performance of commodity printheads is one of factors limiting the expansion of the market. This is a vicious circle in advancing the use of ink jet for printed electronics. The points and pathway to break away from this circle are described bellow.

\subsection{Liquid latitude}

The typical and severe limitation in applying ink jet printhead to printed electronics is liquid latitude. Figure 2

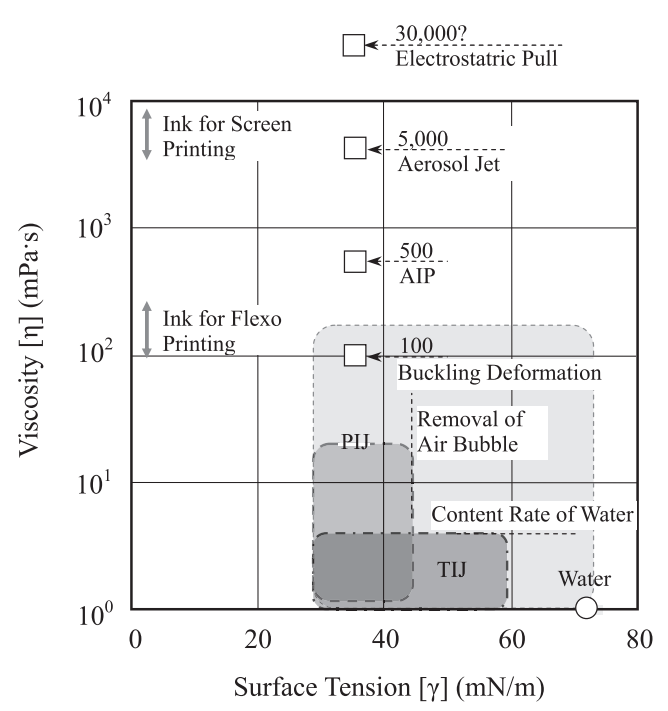

Fig. 2 Liquid latitude handled in ink jet. shows the range of the physical properties of the liquids (viscosity and surface tension) that ink jet can handle.

As to viscosity, the upper limit which a commercial PIJ printhead can eject is approximately $20 \mathrm{mPa} \cdot \mathrm{s}$. If it is necessary to handle liquids over $20 \mathrm{mPa} \cdot \mathrm{s}$, the liquid must be heated to lower the viscosity below the upper limit. This viscosity limitation is a product of the fluid resistance generated by the passage of the liquid in narrow paths and the nozzle. High resistance affects the liquid refill speed due to capillary force and ejection from a narrow nozzle. One approach to ejecting high-viscosity liquids is to get high energy to push the liquid or eject small drops from a large nozzle with low resistance.

In the field of conventional PIJ, a new printhead that generates high PZT power and efficiently transmits it to the liquid has been released. This printhead can eject a $200 \mathrm{mPa} \cdot \mathrm{s}$ liquid without heating; however, it has only single nozzle.

Productivity using an ink jet is to a great extent dominated by printhead specifications. The $S F$ (Speed Factor) expresses how fast an ink jet printhead can print and is given by

$$
S F=\frac{f \cdot N_{z}}{P a s \cdot R_{p} \cdot C r}-T_{m}-X
$$

where $f, N_{z}, P a s, R_{p}$ and $\mathrm{Cr}$ are the cyclic frequency of ejection, number of nozzles, multi-pass number of printing, image resolution and the scanning direction factor, respectively.[3] $T_{m}$ is the time needed for maintenance under printing, and $X$ means other factors (e.g., data-translation time). Drive frequency and nozzle number are important factors for productivity. Improving this new PIJ printhead with multi-nozzles is necessary to enhance productivity in manufacturing.

Aerosol Jet ${ }^{\circledR}$, which generates a forced gas flow to the substrate and blows out mists of highly viscous liquid with this gas has been put to practical use.[4] Aerosol Jet can operate with liquids of roughly $5,000 \mathrm{mPa} \cdot \mathrm{s}$ and has progressed to employing multi-nozzle printheads. However, a mechanical system is used to switch ejecting on and off, so cyclic frequency of ejection is low $(0.5-2 \mathrm{kHz})$. The frequency must be increased tenfold to match other ink jet.

Acoustic Ink Jet (AIP) and Electrostatic Pull Ink Jet are old-fashioned types but can generate small drops from a relatively large nozzle (aperture or open edge of capillary size). These ink jet can also work with liquids of over $500 \mathrm{mPa} \cdot \mathrm{s},[5,6]$ but printheads with multiple nozzles have not been developed yet. AIP and Electrostatic Pull were 
originally developed for printer applications but have not succeeded there because the control and drive of the printhead was complicated and the printer was large for personal or office use. If a printhead with multiple nozzles is developed and productivity is increased, these types will be able feature prominently in printed electronics. The acceptable size and cost must be more moderate than those of a consumer printer.

Thermal Ink Jet (TIJ) printers are commonly used in commercial applications. But TIJ must make vapor bubbles by heating the liquid in order to eject the ink drops. Therefore, TIJ can't handle a wide range of liquids, being limited to aqueous liquids; this is disadvantageous in terms of the degree of freedom in available liquids.

To put printed electronics using ink jet into practical use, a hybrid process called Digital Photolithography, in which an ink jet replaces parts of the process of conventional photolithography, has been the subject of recent research. This research has focused on using an ink jet to eject photosensitive resist or etchant, and a printhead with resistance to strongly alkali or strongly acid liquids was announced.[7] This printhead avoided using adhesives to join materials that contact the liquid by, for example, using screws as much as possible to develop this tolerance.

\subsection{Miniaturization of liquid drop}

It is strongly required to miniaturize the ejected liquid drop to make finer patterns and lines on the substrate in order to improve device performance. As described in the section on liquid latitude, in the situation where a commodity printhead has been used, the minimum liquid drop volume is $1 \mathrm{pl}\left(10^{-12} \mathrm{l}\right)$, which corresponds approximately 20$30 \mu \mathrm{m}$ of dot size on the substrate. It has been reported that a PIJ printhead can decrease the drop volume to $0.3 \mathrm{pl}$ by decreasing the nozzle size.[8] Significant miniaturization with a two-orders-of-magnitude improvement entails solving the problem of large meniscus surface tension at a fine orifice, fluid resistance in a small nozzle, and air resistance, and requires fundamental design changes in the printhead or ejecting principle. For example, liquid drops gain kinetic energy only during ejection, and decelerate due to air resistance (inertia resistance and viscous resistance). This air resistance effect becomes more serious as the drops become smaller; at worst, the drops can't reach the substrate. And drop flight trajectory is even more sensitive to airflow generated by a moving printhead or substrate and dot-positioning errors become larger.

To consider the effects of airflow on the drop trajectory, I have built up a model in which the ink jet printhead faces

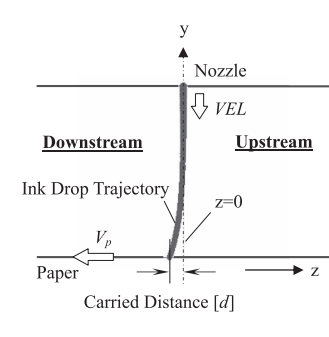

(a)

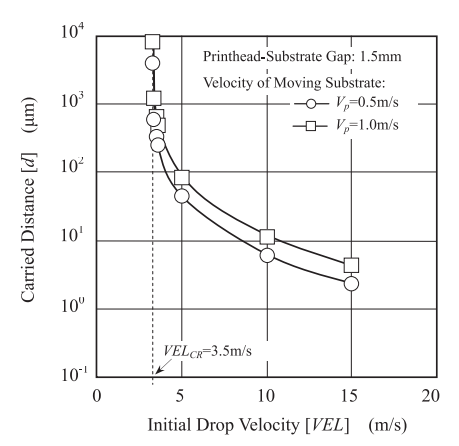

(b)
Fig. 3 Distance that single ink drop ( $0.9 \mathrm{pl})$ was carried by airflow.[9]

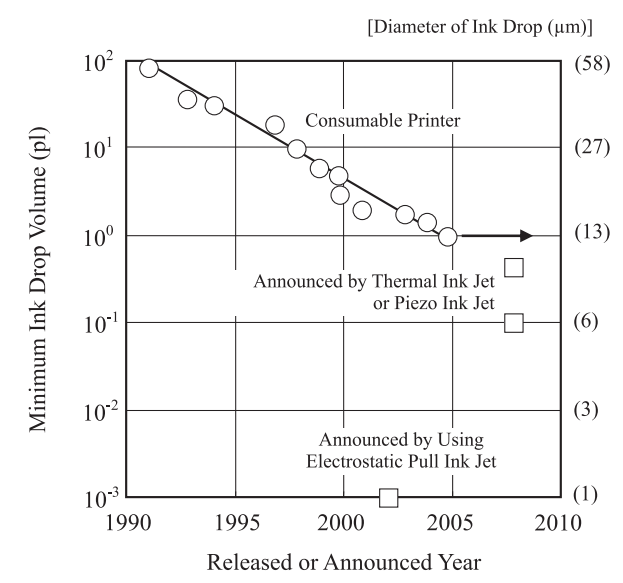

Fig. 4 Trend of minimum drop volume in consumable ink jet printer.[1]

a substrate $1.5 \mathrm{~mm}$ away and calculated the behavior of the drops (Figure 3 (a)). Figure 3 (b) shows the distance $d$ that a liquid drop $(0.9 \mathrm{pl})$ was carried by airflow in the direction of substrate translation while the drop flies for $1.5 \mathrm{~mm}$ (from nozzle to substrate) under the condition of a moving substrate with constant velocity $V_{p}$. [9] The drop trajectory is deflected by the couette flow generated between the printhead and the substrate. A dotted asymptotic line indicates the limit beyond which the drop couldn't reach the substrate. To overcome the effect of air resistance or airflow, it is necessary to increase initial drop velocity $V E L$, to maintain the initial velocity without deceleration, or to remove the air. However, achieving a high initial velocity (for example, over $15 \mathrm{~m} / \mathrm{s}$ in PIJ) or ejecting drops in a vacuum space is a distant goal.

Electrostatic Pull-type ink jet (already discussed above) accelerates charged drops after ejecting using an electric field which can reduce the effects of the air. A Super Ink Jet (SIJ) based on this principle succeeded in ejecting $1 \mathrm{fl}$ $\left(10^{-15}\right)$ drops, [10] which is smaller than commercial ink jet printers by three orders of magnitude. 
Figure 4 shows the trend of minimum drop volume in consumer ink jet printers using PIJ or TIJ technologies.[1] To create small drops in the PIJ and TIJ designs, the nozzle and fluid path must be made narrow. The PIJ and TIJ designs can't add any energy to the liquid drops after they are ejected. From the above-mentioned accounts, PIJ or TIJ printers leap ahead of the current trend significantly and it is impossible to realize $1 \mu \mathrm{m}$ line by extension of the existing trends of consumer printers.

\section{Drop Landing and Dot (Film) Formation}

Paper (cellulose) has been tried as a substrate aiming at cost reduction or weight saving. In most cases using paper, a coating layer is formed on the surface to prevent penetration of the liquid, and patterning caused by penetration of the liquid into the substrate is rarely seen. Of course impermeable substrates (film, glass, silicon, etc.) are often used in printed electronics. Therefore, unlikely conjunctions of dots (e.g., bulges) and inhomogeneous distribution of functional material (e.g., coffee rings) occurring in the drying process on impermeable substrate cause difficulties. Both cases are tightly linked to the wettability of the substrate surface and to the drying environment,[11] and are complicated issues. One approach to getting a desirable pattern shape is forming adjacent dots with a moderate time lag. Also, "multi-pass printing" used in serial commercial ink jet printers has been tried, and produced an improvement. But as shown by Equation (1), this method takes more time to print.

Another attempt which has often been made is to form hydrophilic (or hydrophobic) patterns on the substrate and apply aqueous liquid to the hydrophilic area. An additional process using a photo-mask to differentiate wettability is needed in this method, though self-compensation of dot positioning holds promise if the drop-landing point moves a little. Embrocation of an OLED-emitting material and wiring on the backplane of e-paper has been reported using this process. [12, 13]

Figure 5 shows a new trial I have made with selfcompensation for dot position. In this case, a hydrophilic area was formed on a glass substrate by ejecting fluorinated liquid from an ink jet printhead. UV curable liquid for a micro-lens was also ejected using an ink jet at a slightly different position from the pattern of fluorinated liquid to confirm compensation. If the impact position error was within half a dot, the dot moves spontaneously; that is, self-compensation occurs. This method of using differences in surface energy with an ink jet is useful because

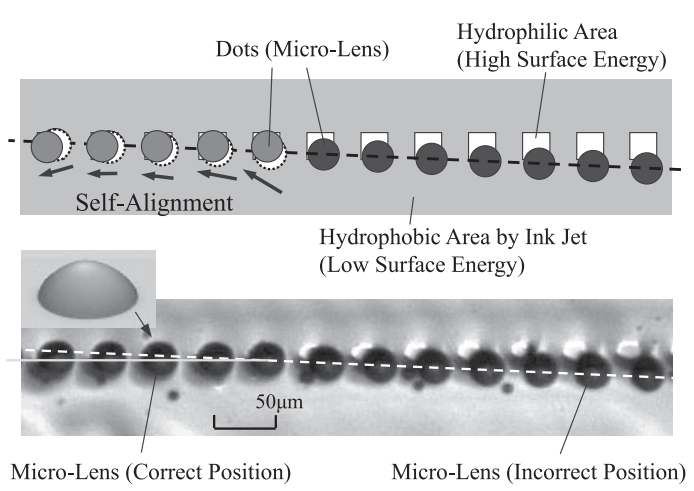

Fig. 5 Self-alignment utilizing differentiation of surface energy.

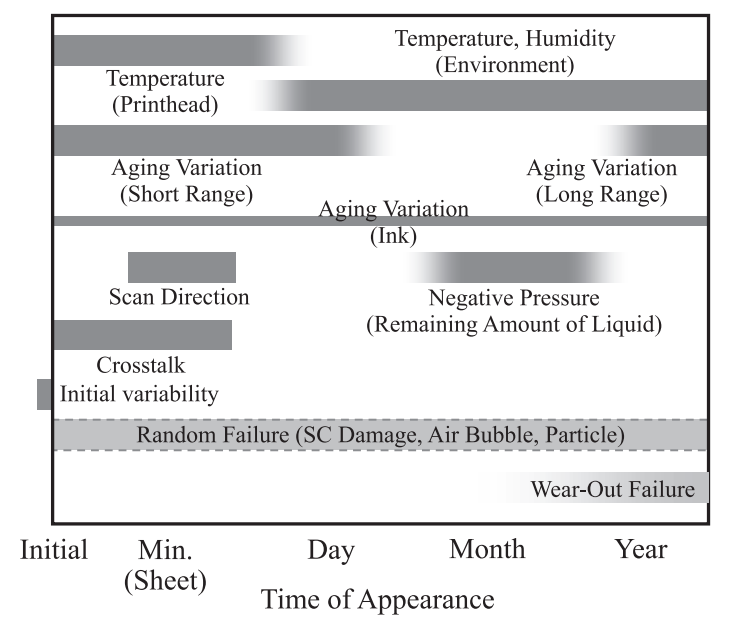

Fig. 6 Factors of performance variation and time of appearance.

no photo-mask is required.

\section{Maintaining Printhead Performance}

Even though high performance ink jet have been demonstrated experimentally, maintaining this high performance for a long period of time is absolutely necessary if ink jet are to be adopted for manufacturing equipment.

Figure 6 summarizes the factors that generate variations in printhead performance and the time when these factors occur in commercial ink jet printers. [14] This frame can be applied to the printed electronics area and most activities aimed at improving performance are also useful with printers. As can be understood from Figure 6, in both the short and long terms, there are factors affecting printhead performance. Different factors can cause similar problems; for example, drops not firing can be caused by not only air bubbles but also damage to the nozzle surface treatment. However, the proper coping strategy depends on the cause of the trouble. Therefore it is important to understand what factor affects performances and to apply the right cor- 
rective measures (maintenance or compensation) at the right time.

Advanced approaches to keeping performance stable are also required in printed electronics. When an ink jet printer creates an image on paper, one dot missing doesn't result in a serious problem in image quality. In contrast, if one dot is not formed in the circuit, it leads to device failure. Liquid circulation in the printhead is effective to prevent evaporation of volatile solvent from the nozzle or unstable ejection caused by air bubbles. This is applied to only a few printer products but is naturally required for printed electronics. Installing a system for degassing the circulating liquid is also required.

\section{Conclusion}

There are noticeable gaps between current ink jet capabilities and the ideal patterning method required by printed electronics. Over time, current ink jet technologies for printers have achieved the functions expected when the ink jet was developed. I strongly believe that ink jet will also acquire the desired performance in the field of printed electronics and their abilities will be exploited in the future. Therefore, first, we should work on the areas (applications) to which ink jet currently contribute and create new value right now in manufacturing practical products in an effort to grow out of the current vicious circle. Ink jet technology may not realizing everything by itself, but it may be part of the solution, with ink jet integrated with current processes as in Digital Photolithography. In applications where ultrafine patterning is not necessary, such as photovoltaic cells or displays, Digital Photolithography may be suitable. In these applications, ink jet features such as the contactless process or high adaptivity to large substrates will be applied fully.

I hold the opinion that ink jet experts and professionals who wish to introduce ink jet technologies have to address each area responsibly and join forces. It is also an important key to bring progress and the success to printed electronics.

\section{References}

[1] M. Fujii, "Footstep and Future Prospect of Ink Technology” Journal of the Imaging Society of Japan, Vol. 47, No. 4, pp. 241-250, 2008 (in Japanese).

[2] T. Shimoda, "Current Status and Future Aspect of Technology for Manufacturing of Electronics
Devices by Liquid Process” Proceeding of Imaging Conference Japan 2007, K1, 2007 (in Japanese).

[3] M. Fujii, "Ink Jet” Tokyo Denki University Press, pp. 1-17, p. 48, 2008 (in Japanese).

[4] B. H. King, "Aerosol Jet ${ }^{\circledR}$ Printing System for High Speed, Non-contact, Front Side Metallization Helping Improve Efficiencies of Silicon Solar Cells,” Proceeding of 24th International Conference on Digital Printing Technologies, pp. 918-922, 2008.

[5] H. Kawamoto, "Fundamental Investigation on Electrostatic Ink Jet Phenomena in Pin-to-Plate Discharge System,” Journal of Imaging Science and Technology, Vol. 49, No. 1, pp. 19-27, 2005.

[6] I. Amemiya, "LED Packaging by Ink-jet Microdeposition of High-Viscosity Resin and Phosphor Dispersion," SID International Symposium Digest of Technical Papers, Vol. 38, No. 2, pp. 1603-1606, 2007.

[7] T. Chen, "Inert Piezoelectric Inkjet Print Head Technology for Alkaline Etch Process in Solar Cell Fabrication," Proceeding of Digital Fabrication 2009, pp. 635-638, 2009.

[8] K. Ozawa, "Development of a Femtoliter Piezo Inkjet Head for high resolution printing,” Proceeding of 23th International Conference on Digital Printing Technologies, pp. 898-901, 2007.

[9] M. Fujii, “Analysis on Behavior of Small Ink Drops Ejected from Ink Jet Printhead,” Journal of the Imaging Society of Japan, Vol. 48, No. 4, pp. 244-254, 2009 (in Japanese).

[10] K. Murata, "Super-fine ink-jet printing: toward the minimal manufacturing system," Microsystem Technologies, Vol. 12, pp. 2-7, 2005.

[11] T. Kajiya, "Piling-to-buckling transition in the drying process of polymer solution drop on substrate having a large contact angle,” Physical Review E, Vol. 73, 011601, 2006.

[12] K. Morii, "Film Formation by Inkjet," Journal of The Surface Science Society of Japan, Vol. 24, No. 2, pp. 90-97, 2003 (in Japanese).

[13] K. Suzuki, "A 160 ppi All-printed Organic TFT Backplane for Flexible Electrophoretic Displays," Proceeding of IDW '08, Vol. 2, pp. 1477-1478, 2008.

[14] M. Fujii, "Collection of the newest ink jet knowhow,” Technical Information Institute, pp. 161, 2005 (in Japanese). 